\title{
MULTI-MEGAWATT W-BAND RF SOURCE BASED ON GYROHARMONIC CONVERSION AT THE EIGHTH HARMONIC*
}

\author{
Changbiao Wang 1 , J. L. Hirshfield ${ }^{1,2}$, and O. A. Nezhevenko ${ }^{2}$ \\ 1Physics Department, Yale University, New Haven, Connecticut 06520-1820 \\ ${ }^{2}$ Omega-P, Inc., 202008 Yale Station, New Haven, Connecticut 06520
}

\section{Abstract}

A new version of an eighth-harmonic converter is analysed, which consists of a $T E_{111}$-mode cavity and an adjacent $T E_{81}$-mode cylindrical waveguide. Driven by 40 MW of rf power at $11.424 \mathrm{GHz}$, a $500 \mathrm{kV}, 60.5 \mathrm{~A}$ Brillouin beam is accelerated in the cavity and generates up to $13 \mathrm{MW}$ of output power at $91.392 \mathrm{GHz}$.

\section{INTRODUCTION}

Gyroharmonic conversion as a process for efficient production of high-power radiation has been discussed in a number of publications [1-6] and various approaches have been proposed. In one approach [1], low frequency rf drive power is used to accelerate an electron beam using the $T E_{11}$ mode in a cylindrical waveguide by means of cyclotron autoresonance acceleration (CARA). The beam then passes through a transition region into a converter section, where it can selectively emit coherent radiation at a harmonic of the drive frequency. To allow the accelerated beam from CARA to fulfill the synchronous and grazing conditions in the converter section, the magnetic field in the transition region is tapered up. The operating harmonic index is specified by proper choice of converter circuit parameters and magnetic field profile. One deficiency in this approach is degradation in beam quality when the beam passes through the transition region where trapping in the strong rf fields is absent.

Another approach to gyroharmonic conversion is cogeneration [5,6], which exploits a near-degeneracy for cylindrical waveguide, where nearly equal phase and group velocities prevail for the $T E_{11}$ mode at frequency $\omega$ and the $T E_{72}$ mode at frequency $7 \omega$. As a result, gyroresonant interactions in the two modes require essentially the same resonant magnetic field profile. Thus efficient transfer of rf energy from the fundamental to the seventh-harmonic can occur in the self-same structure. The harmonic index is selected by the synchronous interaction mechanism itself, instead of through the circuit parameters. The near-degeneracy in cylindrical waveguide referred to above does not exist for an eighthharmonic $T E$-mode interaction.

A new approach to eighth-gyroharmonic conversion is described here. Here the CARA drive section uses a $T E_{111}$-mode cavity and the converter section uses a $T E_{81}$ waveguide where the $T E_{11}$ mode is cutoff and the resonance with the eighth-harmonic is maintained by a proper choice of waveguide radius. Accordingly, no

* The work was supported by the U. S. Department of Energy, Divisions of High Energy Physics and Advanced Energy Projects. transition region is required between the CARA section and the converter section. A cavity is shorter than a waveguide would be for similar beam and rf parameters, and does not require a terminating absorber; both features help in preserving good beam quality.

In this paper, we present preliminary simulation results for the eighth-harmonic converter.

\section{BASIC PRINCIPLES}

Conditions for sustaining resonance for a beam at the end of CARA and the beginning of eighth-harmonic converter section are given by

$$
\omega=\frac{\Omega_{1}}{\gamma_{1}}+k_{z 1} c \beta_{z 1}, \text { and } 8 \omega=\frac{8 \Omega_{2}}{\gamma_{2}}+k_{z 2} c \beta_{z 2},
$$

where the electron gyrofrequency is $\Omega=e B_{0} / m$ with $e$ the electron charge in magnitude, $m$ the rest mass, and $B_{0}$ the axial magnetic field, $k_{z}$ is the axial wavenumber, and the relativistic energy factor is $\gamma=\left(1-\beta_{\perp}^{2}-\beta_{z}^{2}\right)^{-1 / 2}$ with $\beta_{\perp}$ and $\beta_{z}$ the transverse and axial velocities normalized to the vacuum light speed $c$. The parameters with subscript 1 are for the end of CARA and 2 for the beginning of converter section. Since the eighthharmonic output section is directly adjacent to the CARA cavity, the continuity of magnetic field, particle energies, and velocities (that is, $\Omega_{1}=\Omega_{2}, \gamma_{1}=\gamma_{2}$, and $\beta_{z 1}=\beta_{z 2}$ ) requires $8 k_{z 1}=k_{z 2}$. From dispersion equations for $T E_{111}$ and $T E_{81}$ modes, we obtain

$$
\frac{R_{2}}{R_{1}}=\frac{j_{81}^{\prime}}{8 j_{11}^{\prime}}=0.65498,
$$

where $R_{1}$ and $R_{2}$ are, respectively, the $T E_{111}$-cavity radius and $T E_{81}$-waveguide radius, and $j_{11}^{\prime}=1.84118$ and $j_{81}^{\prime}=9.64742$ are the Bessel function roots. It can be noted that an equation similar to the above, but for the $T E_{72}$ mode at frequency $7 \omega$ could be nearly satisfied with $R_{1}=R_{2}$, since $j_{72}^{\prime} / 7=1.84748$; this fact is responsible for the near-degeneracy mentioned previously that can make seventh-harmonic co-generation in the CARA waveguide an efficient process.

Eq. (2) insures that a beam exiting the $T E_{111}$-mode cavity at frequency $\omega$ remains resonant with the $T E_{81}$ mode at frequency $8 \omega$. According to selection rules for axisymmetric beams [2], $T E_{11}$ mode at the fundamental is the most competitive to the $T E_{81}$ mode in the converter section. It can be shown that a proper choice of the cavity radius $R_{1}$ insures that the $T E_{11}$ mode at the fundamental (or the $T E_{72}$ mode at the seventh harmonic) is cutoff. If the fundamental frequency $f_{1}$ is $11.424 \mathrm{GHz}$ 
$\left(\lambda_{1}=2.626 \mathrm{~cm}\right)$, and the eighth-harmonic frequency $f_{8}$ is $91.392 \mathrm{GHz}\left(\lambda_{8}=0.328 \mathrm{~cm}\right)$. Taking the cavity radius $R_{1}=0.798 \mathrm{~cm}$, from Eq. (2) we have the converter section radius $R_{2}=0.522 \mathrm{~cm}$. The cutoff wavelengths of $T E_{11}$ mode at $f_{1}$ and $T E_{81}$ mode at $f_{8}$ are, respectively, $1.783 \mathrm{~cm}$ and $0.340 \mathrm{~cm}$, which means that $T E_{81}$ is a propagating mode while $T E_{11}$ is cutoff.

\section{SIMULATION RESULTS}

A preliminary simulation study has been performed for this harmonic converter configuration. The design parameters are chosen to accommodate as initial conditions at the CARA entrance a Brillouin beam. The beam radius $r_{b}$, beam current $I$, guide magnetic field $B_{0}$, and beam energy factor $\gamma_{0}$ are related by [7]

$$
r_{b}=\frac{1}{B_{0}}\left[\frac{2 m}{\pi \varepsilon_{0} e c} \frac{I}{\left(\gamma_{0}^{2}-1\right)^{1 / 2}}\right]^{1 / 2} .
$$

For the example that was analyzed, a $500 \mathrm{kV}, 60.5 \mathrm{~A}$ Brillouin beam with a radius of $0.35 \mathrm{~mm}$ is injected along the axis of a $T E_{111}$ CARA cavity with a radius of 0.798 $\mathrm{cm}$ and a length of $4.95 \mathrm{~cm}$. The cavity resonates at $11.424 \mathrm{GHz}$ and is driven with a power of $40 \mathrm{MW}$; the unloaded cavity quality factor $Q_{0}$ is 5000 . The $T E_{81}{ }^{-}$ mode output waveguide has a radius of $0.522 \mathrm{~cm}$ and a length of $3.0 \mathrm{~cm}$, following which a gentle up-taper in wall radius accompanies a linear down-taper to zero in guide magnetic field, allowing the beam to be collected on the wall. The simulation parameters are given in Table I.

Table I: Parameters in simulation

\begin{tabular}{ll}
\hline Beam energy & $500 \mathrm{keV}$ \\
Beam current & $60.5 \mathrm{~A}$ \\
Beam radius & $0.35 \mathrm{~mm}$ \\
Input rf power & $40 \mathrm{MW}$ \\
Drive frequency & $11.424 \mathrm{GHz}$ \\
$T E_{111}$ cavity radius $R_{1}$ & $0.798 \mathrm{~cm}$ \\
Cavity length $L$ & $4.95 \mathrm{~cm}$ \\
Unloaded cavity quality factor $Q_{0}$ & 5000 \\
Loaded cavity quality factor $Q_{L}$ & 100 \\
$T E_{81}$ waveguide radius $R_{2}$ & $0.522 \mathrm{~cm}$ \\
$T E_{81}$ waveguide length & $3 \mathrm{~cm}$ \\
Final collector radius & $0.6 \mathrm{~cm}$ \\
Collector length & $10 \mathrm{~cm}$ \\
\hline
\end{tabular}

Fig. 1 shows the rf structure outline and orbits of 8 sample particles in the $r-z$ plane, and Fig. 2 shows the relativistic energy factors for the particles. In the CARA section (from $z=0$ to $4.95 \mathrm{~cm}$ ), the electrons are accelerated and their energies are increased. In the output section including the $T E_{81}$ waveguide and collector (from $z=4.95$ to $17.95 \mathrm{~cm}$ ), the electrons lose energy to $T E_{81}$ mode at the eighth harmonic and then the wall intercepts all the electrons, because the axial magnetic field is linearly reduced to zero between $z=7.95$ and $17.95 \mathrm{~cm}$.

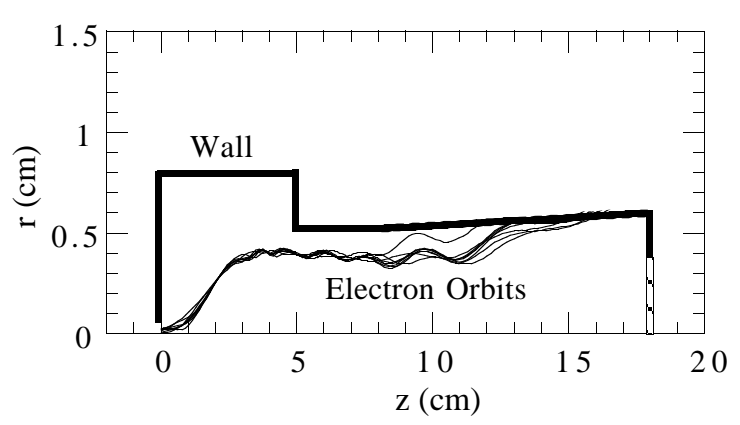

Figure 1: Wall profile for CARA cavity, output waveguide and tapered collector, and orbits throughout device for 8 sample electrons of 512 taken in simulation, including those orbits with extreme excursions.

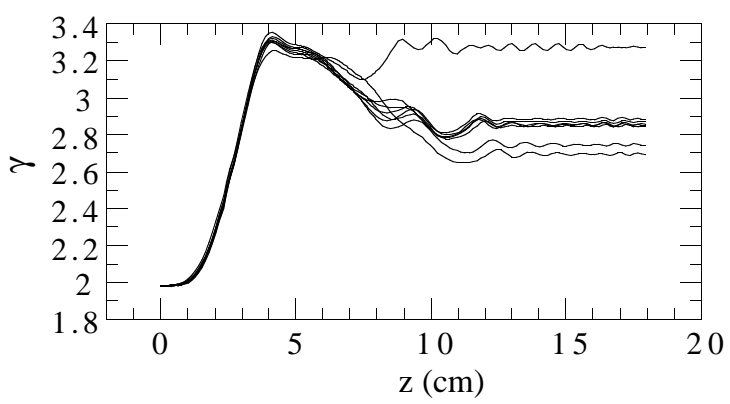

Figure 2: Energy factors for the 8 electrons in Fig. 1.

Fig. 3 shows the rf power levels and axial magnetic field profile along the full $17.95-\mathrm{cm}$ length of the device. With a power of $40 \mathrm{MW}$ at $11.424 \mathrm{GHz}$, the beam is accelerated initially from $500 \mathrm{keV}$ to $1.15 \mathrm{MeV}$ at the end of the cavity; this corresponds to an acceleration efficiency of $98 \%$ and an loaded cavity quality factor of 100 ; the magnetic field varies from 6.2 to $12.5 \mathrm{kG}$. In the output section, the eighth-harmonic rf power at $91.392 \mathrm{GHz}$ is gradually increased and then reaches a maximum of 14.9 MW at $z=11 \mathrm{~cm}$. Because of wall loss after about $z=13 \mathrm{~cm}$ where no net energy exchange takes place, the power is slightly reduced with a final output power of 13.1 MW. The magnetic field is resonantly decreased from $11.6 \mathrm{kG}(z=4.95 \mathrm{~cm})$ to $10.3 \mathrm{kG}(z=7.95 \mathrm{~cm})$, and then linearly to zero $(z=17.95 \mathrm{~cm})$.

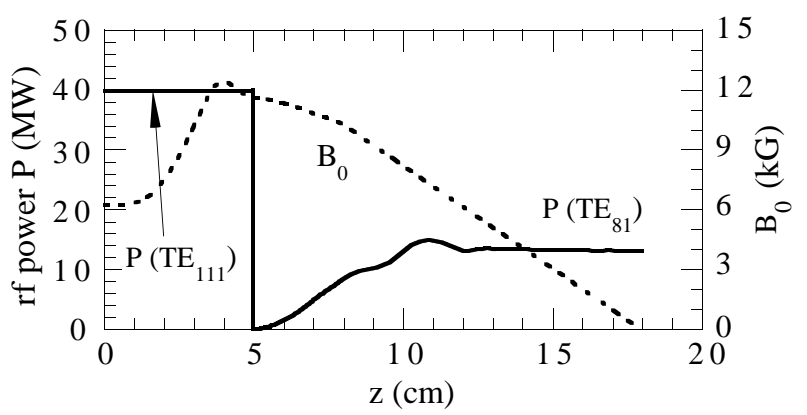

Figure 3: rf power levels in CARA and in output section (solid line), and magnetic field profile (dashed line). 
Peak field strengths and ohmic power loss in the structure have been computed as well, and have been found to be well below the accepted levels for carefully built high power structures at X-band and W-band [8]. Results of these computations are shown in Figs. 4 and 5.

Fig. 4 shows the dependence of peak radial rf field strength at the walls in CARA and output section on axial distance. The maximum electric field in the CARA is $0.326 \mathrm{MV} / \mathrm{cm}$ and $0.266 \mathrm{MV} / \mathrm{cm}$ in the output section, well below the accepted breakdown limits for copper with $1-2 \mu$ sec pulses [9].

Fig. 5 shows the dependence of peak wall loss power $P_{\text {wall }}$ and power density $d P / d A$ on axial distance $z$ for the output waveguide. Note that for a duty cycle less than $10^{-3}$, the maximum average power density to be dissipated at the walls of the output waveguide would be less than $30 \mathrm{~W} / \mathrm{cm}^{2}$. The cavity must dissipate an average power of $800 \mathrm{~W}$ at this duty cycle.

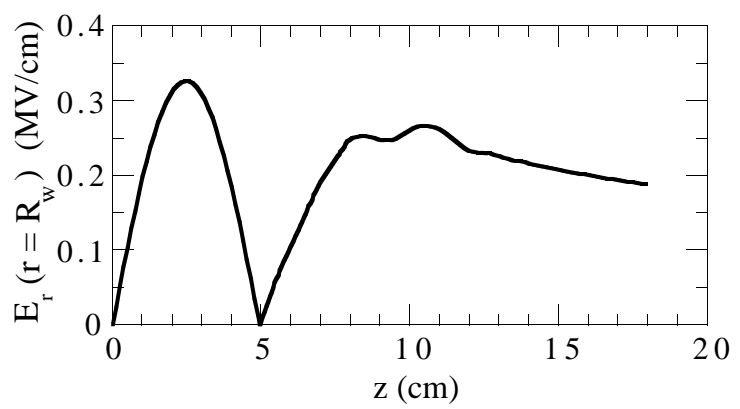

Figure 4: Peak radial rf field strength at the walls in CARA and in the output waveguide.

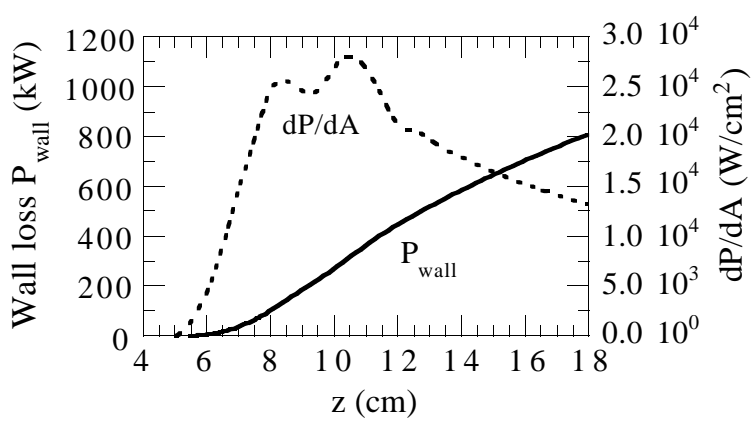

Figure 5: Cumulative peak power (solid line) and power density to be dissipated on the walls of the output waveguide (dashed line).

\section{REMARKS}

By simulation, we have analyzed an eighth-harmonic converter which uses a $T E_{111}$-mode cavity as the drive section and a $T E_{81}$-waveguide as the output section, without any required transition region. With an input power of $40 \mathrm{MW}$ at $11.424 \mathrm{GHz}$ and a $0.35-\mathrm{mm}$ radius, 500-kV, 60.5-A Brillouin beam, a net output power of 13 MW at $91.392 \mathrm{GHz}$ is obtained. As beam current and beam radius increase, the efficiency for eighth-harmonic conversion falls, although the absolute magnitude of output power can increase; likewise, an increase in rf drive power level can be tolerated at higher current, thereby also making higher output power possible.

Mode competition which is not taken into account in the simulation can occur in an overmoded rf structure as used in such a harmonic converter. In the CARA cavity, co-generation of seventh-harmonic power could arise, should it turn out that one of the $T E_{72 m}$ modes of the cavity were to resonate at $79.968 \mathrm{GHz}$. For a perfect cylinder with $R_{1}=0.798 \mathrm{~cm}, L=4.95 \mathrm{~cm}$, probably the most dangerous (i. e., nearest) eigenmode is the $T E_{727}$, whose eigenfrequency is $80.222 \mathrm{GHz}$. But if the loaded $Q$ for the $T E_{727}$ mode is much larger than $80.222 / 2(80.222-79.968)=158$, it should be possible to avoid excitation of the seventh harmonic because of detuning. Mode competition might also pose a problem in the output waveguide, since the $T E_{61}$ and $T E_{71}$ modes are not cutoff at the sixth and seventh harmonics. In the extreme, it is possible to operate with a cavity in place of the output waveguide, in order to discriminate against frequencies other than the design frequency of 91.392 GHz. In a preliminary simulation for a $T E_{811}$-mode output cavity, whose ohmic $Q$ for copper cannot be higher than about 5000, and for the beam parameters and $40 \mathrm{MW}-11.424 \mathrm{GHz}$ drive level used in Figs. 1-5, a power of $12.06 \mathrm{MW}$ is obtained. But $4.28 \mathrm{MW}$ of this is dissipated in wall losses in the output cavity where $Q_{L}=1774$; so the net output is $7.78 \mathrm{MW}$. These considerations suggest that strategies to avoid serious mode competition, and to operate insofar as possible without undue ohmic wall losses, will probably be the major challenges for further investigations.

\section{REFERENCES}

[1] J. L. Hirshfield, C. Wang, and A. K. Ganguly, IEEE Trans. Plasma Sci. PS-24, 825 (1996).

[2] A. K. Ganguly and J. L. Hirshfield, Phys. Rev. E 47, 4364 (1993).

[3] A. J. Balcum, D. B. McDermott, K. C. Leou, F. V. Hartmann, and N. C. Luhmann, Jr., IEEE Trans. Plasma Sci., PS-22, 913 (1994).

[4] H. R. Jory and A. W. Trivelpiece, J. Appl. Phys. 39, 3053 (1968).

[5] C. Wang, J. L. Hirshfield, and A. K. Ganguly, Phys. Rev. Lett. 77, 3819 (1996).

[6] J. L. Hirshfield, C. Wang, and A. K. Ganguly, IEEE Trans. Plasma Sci., PS-26, 567 (1998).

[7] M. Reiser, Theory and Design of Charged Particle Beams (Wiley, New York, 1994).

[8] J. W. Wang and G. A. Loew, SLAC-PUB-7684, Stanford University (1997).

[9] D. G. Myakishev, M. A. Tiunov, and V. P. Yakovlev, Int. J. Mod. Phys. A, 915 (1993). 